\title{
Search for $B_{s}^{0} \rightarrow \mu^{+} \mu^{-}$and $B_{d}^{0} \rightarrow \mu^{+} \mu^{-}$Decays in $p \bar{p}$ Collisions with CDF II
}

A. Abulencia, ${ }^{23}$ D. Acosta, ${ }^{17}$ J. Adelman, ${ }^{13}$ T. Affolder, ${ }^{10}$ T. Akimoto, ${ }^{53}$ M. G. Albrow, ${ }^{16}$ D. Ambrose, ${ }^{16}$ S. Amerio, ${ }^{42}$ D. Amidei, ${ }^{33}$ A. Anastassov, ${ }^{50}$ K. Anikeev,${ }^{16}$ A. Annovi, ${ }^{44}$ J. Antos, ${ }^{1}$ M. Aoki, ${ }^{53}$ G. Apollinari, ${ }^{16}$ J.-F. Arguin, ${ }^{32}$

T. Arisawa, ${ }^{55}$ A. Artikov, ${ }^{44}$ W. Ashmanskas, ${ }^{16}$ A. Attal, ${ }^{8}$ F. Azfar, ${ }^{41}$ P. Azzi-Bacchetta, ${ }^{42}$ P. Azzurri, ${ }^{44}$ N. Bacchetta, ${ }^{42}$ H. Bachacou, ${ }^{28}$ W. Badgett, ${ }^{16}$ A. Barbaro-Galtieri, ${ }^{28}$ V. E. Barnes, ${ }^{46}$ B. A. Barnett, ${ }^{47}$ S. Baroiant,${ }^{7}$ V. Bartsch,${ }^{30}$ G. Bauer,${ }^{31}$ F. Bedeschi, ${ }^{44}$ S. Behari, ${ }^{24}$ S. Belforte,${ }^{52}$ G. Bellettini, ${ }^{44}$ J. Bellinger,${ }^{57}$ A. Belloni, ${ }^{31}$ E. Ben-Haim, ${ }^{16}$ D. Benjamin, ${ }^{15}$ A. Beretvas, ${ }^{16}$ J. Beringer, ${ }^{28}$ T. Berry, ${ }^{29}$ A. Bhatti, ${ }^{48}$ M. Binkley, ${ }^{1}$ D. Bisello, ${ }^{42}$ M. Bishai, ${ }^{16}$ R. E. Blair, ${ }^{2}$ C. Blocker, ${ }^{6}$ K. Bloom ${ }^{33}$ B. Blumenfeld ${ }^{24}$ A. Bocci, ${ }^{48}$ A. Bodek,${ }^{47}$ V. Boisvert,${ }^{47}$ G. Bolla ${ }^{46}$ A. Bolshov,${ }^{31}$ D. Bortoletto, ${ }^{46}$ J. Boudreau, ${ }^{44}$ S. Bourov,${ }^{16}$ A. Boveia,${ }^{10}$ B. Brau,${ }^{10}$ C. Bromberg, ${ }^{34}$ E. Brubaker, ${ }^{13}$ J. Budagov, ${ }^{14}$ H. S. Budd, ${ }^{47}$ S. Budd ${ }^{23}$ K. Burkett, ${ }^{16}$ G. Busetto, ${ }^{42}$ P. Bussey, ${ }^{20}$ K. L. Byrum, ${ }^{2}$ S. Cabrera, ${ }^{15}$ M. Campanelli, ${ }^{19}$ M. Campbell, ${ }^{33}$ F. Canelli, ${ }^{8}$ A. Canepa,${ }^{46}$ D. Carlsmith, ${ }^{57}$ R. Carosi,${ }^{44}$ S. Carron, ${ }^{15}$ M. Casarsa,${ }^{52}$ A. Castro, ${ }^{5}$ P. Catastini, ${ }^{4}$ D. Cauz,${ }^{52}$ M. Cavalli-Sforza, ${ }^{33}$ A. Cerri, ${ }^{28}$ L. Cerrito, ${ }^{41}$ S. H. Chang, ${ }^{27}$ J. Chapman, ${ }^{33}$ Y. C. Chen, ${ }^{1}$ M. Chertok, ${ }^{7}$ G. Chiarelli, ${ }^{44}$ G. Chlachidze, ${ }^{14}$ F. Chlebana, ${ }^{16}$ I. Cho, ${ }^{27}$ K. Cho, ${ }^{27}$ D. Chokheli, ${ }^{14}$ J. P. Chou,${ }^{21}$ P. H. Chu, ${ }^{23}$ S. H. Chuang, ${ }^{57}$ K. Chung, ${ }^{18}$ W. H. Chung, ${ }^{57}$ Y. S. Chung, ${ }^{47}$ M. Ciljak, ${ }^{44}$ C. I. Ciobanu, ${ }^{23}$ M. A. Ciocci, ${ }^{44}$ A. Clark, ${ }^{19}$ D. Clark, ${ }^{6}$ M. Coca, ${ }^{15}$ A. Connolly, ${ }^{28}$ M. E. Convery, ${ }^{48}$ J. Conway, ${ }^{7}$ B. Cooper,${ }^{39}$ K. Copic,${ }^{33}$ M. Cordelli, ${ }^{18}$ G. Cortiana, ${ }^{42}$ A. Cruz, ${ }^{17}$ J. Cuevas, ${ }^{11}$ R. Culbertson, ${ }^{16}$ D. Cyr,${ }^{57}$ S. DaRonco, ${ }^{42}$ S. D'Auria, ${ }^{20}$ M. D'onofrio, ${ }^{19}$ D. Dagenhart, ${ }^{6}$ P. de Barbaro, ${ }^{47}$ S. De Cecco, ${ }^{49}$ A. Deisher, ${ }^{28}$ G. De Lentdecker, ${ }^{47}$ M. Dell' Orso, ${ }^{44}$ S. Demers, ${ }^{47}$ L. Demortier ${ }^{48}$ J. Deng, ${ }^{15}$ M. Deninno, ${ }^{5}$ D. De Pedis,${ }^{49}$ P. F. Derwent,${ }^{16}$ C. Dionisi, ${ }^{49}$ J. Dittmann, ${ }^{4}$ P. DiTuro,${ }^{50}$ C. Dörr, ${ }^{25}$ A. Dominguez, ${ }^{28}$ S. Donati, ${ }^{44}$ M. Donega,${ }^{19}$ P. Dong,${ }^{8}$ J. Donini, ${ }^{42}$ T. Dorigo, ${ }^{42}$ S. Dube,${ }^{50}$ K. Ebina, ${ }^{55}$ J. Efron, ${ }^{38}$ J. Ehlers, ${ }^{19}$ R. Erbacher, ${ }^{7}$ D. Errede,${ }^{23}$ S. Errede,${ }^{23}$ R. Eusebi,${ }^{47}$ H. C. Fang, ${ }^{28}$ S. Farrington, ${ }^{29}$ I. Fedorko, ${ }^{44}$ W. T. Fedorko, ${ }^{13}$ R. G. Feild, ${ }^{58}$ M. Feindt, ${ }^{25}$ J. P. Fernandez,${ }^{46}$ R. Field, ${ }^{17}$ G. Flanagan, ${ }^{34}$ L. R. Flores-Castillo, ${ }^{45}$ A. Foland, ${ }^{21}$ S. Forrester, ${ }^{7}$ G. W. Foster, ${ }^{16}$ M. Franklin, ${ }^{21}$ J. C. Freeman, ${ }^{28}$ Y. Fujii, ${ }^{26}$ I. Furic, ${ }^{13}$ A. Gajjar, ${ }^{29}$ M. Gallinaro, ${ }^{48}$ J. Galyardt, ${ }^{12}$ J. E. Garcia, ${ }^{44}$ M. Garcia Sciverez,${ }^{28}$ A. F. Garfinkel, ${ }^{46}$ C. Gay, ${ }^{58}$ H. Gerberich, ${ }^{23}$ E. Gerchtein, ${ }^{12}$ D. Gerdes,${ }^{33}$ S. Giagu, ${ }^{49}$ P. Giannetti, ${ }^{44}$ A. Gibson, ${ }^{28}$ K. Gibson, ${ }^{12}$ C. Ginsburg, ${ }^{16}$ K. Giolo, ${ }^{46}$ M. Giordani, ${ }^{52}$ M. Giunta, ${ }^{44}$ G. Giurgiu, ${ }^{12}$ V. Glagolev, ${ }^{14}$ D. Glenzinski, ${ }^{16}$ M. Gold ${ }^{36}$ N. Goldschmidt,${ }^{33}$ J. Goldstein, ${ }^{41}$ G. Gomez, ${ }^{11}$ G. Gomez-Ceballos, ${ }^{11}$ M. Goncharov, ${ }^{51}$ O. González, ${ }^{46}$ I. Gorelov, ${ }^{36}$ A. T. Goshaw, ${ }^{15}$ Y. Gotra, ${ }^{45}$ K. Goulianos, ${ }^{48}$ A. Gresele, ${ }^{42}$ M. Griffiths,${ }^{29}$ S. Grinstein, ${ }^{21}$ C. Grosso-Pilcher, ${ }^{13}$ U. Grundler,${ }^{23}$ J. Guimaraes da Costa, ${ }^{21}$ C. Haber, ${ }^{28}$ S. R. Hahn, ${ }^{16}$ K. Hahn, ${ }^{43}$ E. Halkiadakis, ${ }^{47}$ A. Hamilton, ${ }^{32}$ B.-Y. Han, ${ }^{47}$ R. Handler, ${ }^{57}$ F. Happacher, ${ }^{18}$ K. Hara, ${ }^{53}$ M. Hare,${ }^{54}$ S. Harper,${ }^{41}$ R. F. Harr,${ }^{56}$ R. M. Harris, ${ }^{16}$ K. Hatakeyama,${ }^{48}$ J. Hauser, ${ }^{8}$ C. Hays, ${ }^{15}$ H. Hayward ${ }^{29}$ A. Heijboer, ${ }^{43}$ B. Heinemann, ${ }^{29}$ J. Heinrich,${ }^{43}$ M. Hennecke, ${ }^{25}$ M. Herndon, ${ }^{57}$ J. Heuser, ${ }^{25}$ D. Hidas, ${ }^{15}$ C. S. Hill, ${ }^{10}$ D. Hirschbuehl, ${ }^{25}$ A. Hocker, ${ }^{16}$ A. Holloway, ${ }^{21}$ S. Hou, ${ }^{1}$ M. Houlden, ${ }^{29}$ S.-C. Hsu, ${ }^{9}$ B. T. Huffman, ${ }^{41}$ R. E. Hughes,${ }^{38} \mathrm{~J}$. Huston, ${ }^{34} \mathrm{~K}$. Ikado, ${ }^{55} \mathrm{~J}$. Incandela, ${ }^{10} \mathrm{G}$. Introzzi, ${ }^{44} \mathrm{M}$. Iori,${ }^{49}$ Y. Ishizawa, ${ }^{53}$ A. Ivanov, ${ }^{7}$ B. Iyutin, ${ }^{31}$ E. James, ${ }^{16}$ D. Jang, ${ }^{50}$ B. Jayatilaka, ${ }^{33}$ D. Jeans, ${ }^{49}$ H. Jensen, ${ }^{16}$ E. J. Jeon,${ }^{27}$ M. Jones, ${ }^{46}$ K. K. Joo, ${ }^{27}$ S. Y. Jun, ${ }^{12}$

T. R. Junk, ${ }^{23}$ T. Kamon, ${ }^{51}$ J. Kang, ${ }^{33}$ M. Karagoz-Unel, ${ }^{37}$ P. E. Karchin, ${ }^{56}$ Y. Kato, ${ }^{40}$ Y. Kemp,${ }^{25}$ R. Kephart, ${ }^{16}$ U. Kerzel, ${ }^{25}$ V. Khotilovich, ${ }^{51}$ B. Kilminster, ${ }^{38}$ D. H. Kim,${ }^{27}$ H. S. Kim, ${ }^{27}$ J.E. Kim, ${ }^{27}$ M. J. Kim, ${ }^{12}$ M. S. Kim, ${ }^{27}$ S. B. Kim, ${ }^{27}$ S. H. Kim,${ }^{53}$ Y. K. Kim,,${ }^{13}$ M. Kirby, ${ }^{15}$ L. Kirsch,${ }^{6}$ S. Klimenko, ${ }^{17}$ M. Klute,${ }^{31}$ B. Knuteson, ${ }^{31}$ B. R. Ko, ${ }^{15}$ H. Kobayashi, ${ }^{53}$

K. Kondo, ${ }^{55}$ D. J. Kong, ${ }^{27}$ J. Konigsberg, ${ }^{17}$ K. Kordas, ${ }^{18}$ A. Korytov, ${ }^{17}$ A. V. Kotwal, ${ }^{15}$ A. Kovalev, ${ }^{43}$ J. Kraus, ${ }^{23}$ I. Kravchenko, ${ }^{31}$ M. Kreps ${ }^{25}$ A. Kreymer, ${ }^{16}$ J. Kroll ${ }^{43}$ N. Krumnack, ${ }^{4}$ M. Kruse, ${ }^{15}$ V. Krutelyov, ${ }^{51}$ S. E. Kuhlmann, ${ }^{2}$ Y. Kusakabe, ${ }^{55}$ S. Kwang, ${ }^{13}$ A. T. Laasanen, ${ }^{46}$ S. Lai, ${ }^{32}$ S. Lami, ${ }^{48}$ S. Lami, ${ }^{48}$ S. Lammel, ${ }^{16}$ M. Lancaster, ${ }^{30}$ R. L. Lander, ${ }^{7}$ K. Lannon, ${ }^{38}$ A. Lath, ${ }^{50}$ G. Latino, ${ }^{44}$ I. Lazzizzera, ${ }^{42}$ C. Lecci, ${ }^{25}$ T. LeCompte,${ }^{2}$ J. Lee,${ }^{47}$ J. Lee,${ }^{47}$ S. W. Lee,${ }^{51}$ R. Lefèvre, ${ }^{3}$ N. Leonardo, ${ }^{31}$ S. Leone, ${ }^{44}$ S. Levy, ${ }^{13}$ J. D. Lewis,${ }^{16}$ K. Li,${ }^{58}$ C. Lin,${ }^{58}$ C. S. Lin,${ }^{16}$ M. Lindgren,${ }^{16}$ E. Lipeles,${ }^{9}$ T. M. Liss,${ }^{23}$ A. Lister, ${ }^{19}$ D. O. Litvintsev, ${ }^{16}$ T. Liu, ${ }^{16}$ Y. Liu, ${ }^{19}$ N. S. Lockyer, ${ }^{43}$ A. Loginov, ${ }^{35}$ M. Loreti, ${ }^{42}$ P. Loverre, ${ }^{49}$ R.-S. Lu, ${ }^{1}$ D. Lucchesi, ${ }^{42}$ P. Lujan, ${ }^{28}$ P. Lukens, ${ }^{16}$ G. Lungu, ${ }^{17}$ L. Lyons, ${ }^{41}$ J. Lys,${ }^{28}$ R. Lysak, ${ }^{1}$ E. Lytken, ${ }^{46}$ P. Mack, ${ }^{25}$

D. MacQueen, ${ }^{32}$ R. Madrak,${ }^{16}$ K. Maeshima,${ }^{16}$ P. Maksimovic,${ }^{24}$ G. Manca, ${ }^{29}$ F. Margaroli, ${ }^{5}$ R. Marginean,${ }^{16}$ C. Marino, ${ }^{23}$ A. Martin, ${ }^{58}$ M. Martin, ${ }^{24}$ V. Martin,${ }^{37}$ M. Martínez, ${ }^{3}$ T. Maruyama,${ }^{53}$ H. Matsunaga,${ }^{53}$ M. E. Mattson,${ }^{56}$ R. Mazini, ${ }^{32}$

P. Mazzanti, ${ }^{5}$ K. S. McFarland,${ }^{47}$ D. McGivern, ${ }^{30}$ P. McIntyre,${ }^{51}$ P. McNamara, ${ }^{50}$ R. McNulty, ${ }^{29}$ A. Mehta, ${ }^{29}$

S. Menzemer ${ }^{31}$ A. Menzione,${ }^{44}$ P. Merkel,${ }^{46}$ C. Mesropian, ${ }^{48}$ A. Messina,${ }^{49}$ M. von der Mey, ${ }^{8}$ T. Miao, ${ }^{16}$ N. Miladinovic,${ }^{6}$ J. Miles, ${ }^{31}$ R. Miller, ${ }^{34}$ J. S. Miller, ${ }^{33}$ C. Mills,,${ }^{10}$ M. Milnik, ${ }^{25}$ R. Miquel,${ }^{28}$ S. Miscetti, ${ }^{18}$ G. Mitselmakher,${ }^{17}$ A. Miyamoto ${ }^{26}$ N. Moggi, ${ }^{5}$ B. Mohr, ${ }^{8}$ R. Moore,${ }^{16}$ M. Morello, ${ }^{44}$ P. Movilla Fernandez, ${ }^{28}$ J. Mülmenstädt, ${ }^{28}$ A. Mukherjee, ${ }^{16}$ M. Mulhearn, ${ }^{31}$ Th. Muller,${ }^{25}$ R. Mumford, ${ }^{24}$ P. Murat,${ }^{16}$ J. Nachtman, ${ }^{16}$ S. Nahn, ${ }^{58}$ I. Nakano, ${ }^{39}$ 
A. Napier, ${ }^{54}$ D. Naumov, ${ }^{36}$ V. Necula, ${ }^{17}$ C. Neu, ${ }^{43}$ M. S. Neubauer, ${ }^{9}$ J. Nielsen, ${ }^{28}$ T. Nigmanov,${ }^{45}$ L. Nodulman, ${ }^{2}$ O. Norniella, ${ }^{3}$ T. Ogawa, ${ }^{55}$ S. H. Oh, ${ }^{15}$ Y. D. Oh,${ }^{27}$ T. Okusawa, ${ }^{40}$ R. Oldeman, ${ }^{29}$ R. Orava, ${ }^{22}$ K. Osterberg, ${ }^{22}$ C. Pagliarone,${ }^{44}$ E. Palencia, ${ }^{11}$ R. Paoletti, ${ }^{44}$ V. Papadimitriou, ${ }^{16}$ A. Papikonomou, ${ }^{25}$ A. A. Paramonov, ${ }^{13}$ B. Parks,${ }^{38}$ S. Pashapour, ${ }^{32}$ J. Patrick, ${ }^{16}$ G. Pauletta, ${ }^{52}$ M. Paulini, ${ }^{12}$ C. Paus,${ }^{31}$ D. E. Pellett, ${ }^{7}$ A. Penzo, ${ }^{52}$ T. J. Phillips, ${ }^{15}$ G. Piacentino, ${ }^{44}$ J. Piedra, ${ }^{11}$ K. Pitts,${ }^{23}$ C. Plager ${ }^{8}$ L. Pondrom,${ }^{57}$ G. Pope,${ }^{45}$ X. Portell,,${ }^{3}$ O. Poukhov,${ }^{14}$ N. Pounder, ${ }^{41}$ F. Prakoshyn, ${ }^{14}$ A. Pronko, ${ }^{16}$ J. Proudfoot, ${ }^{2}$ F. Ptohos, ${ }^{18}$ G. Punzi,${ }^{44}$ J. Pursley, ${ }^{24}$ J. Rademacker,${ }^{41}$ A. Rahaman, ${ }^{45}$ A. Rakitin, ${ }^{31}$ S. Rappoccio, ${ }^{21}$ F. Ratnikov,${ }^{50}$ B. Reisert, ${ }^{16}$ V. Rekovic, ${ }^{36}$ N. van Remortal, ${ }^{22}$ P. Renton, ${ }^{41}$ M. Rescigno, ${ }^{49}$ S. Richter, ${ }^{25}$ F. Rimondi, ${ }^{5}$ K. Rinnert, ${ }^{25}$ L. Ristori ${ }^{44}$ W. J. Robertson, ${ }^{15}$ A. Robson,${ }^{20}$ T. Rodrigo, ${ }^{11}$ E. Rogers, ${ }^{23}$ S. Rolli, ${ }^{54}$ R. Roser, ${ }^{16}$ M. Rossi, ${ }^{52}$ R. Rossin, ${ }^{17}$ C. Rott, ${ }^{46}$ A. Ruiz,${ }^{11}$ J. Russ, ${ }^{12}$ V. Rusu, ${ }^{13}$ D. Ryan, ${ }^{54}$ H. Saarikko, ${ }^{22}$ S. Sabik, ${ }^{32}$ A. Safonov, ${ }^{7}$ W. K. Sakumoto, ${ }^{47}$ G. Salamanna, ${ }^{49}$ O. Salto, ${ }^{3}$ D. Saltzberg, ${ }^{8}$ C. Sanchez, ${ }^{3}$ L. Santi, ${ }^{52}$ S. Sarkar, ${ }^{49}$ K. Sato, ${ }^{53}$ P. Savard, ${ }^{32}$ A. Savoy-Navarro, ${ }^{16}$ T. Scheidle, ${ }^{25}$ P. Schlabach, ${ }^{16}$ E. E. Schmidt,,${ }^{16}$ M. P. Schmidt, ${ }^{58}$ M. Schmitt, ${ }^{37}$ T. Schwarz, ${ }^{33}$ L. Scodellaro, ${ }^{11}$ A. L. Scott, ${ }^{10}$ A. Scribano, ${ }^{44}$ F. Scuri, ${ }^{44}$ A. Sedov ${ }^{46}$ S. Seidel,${ }^{36}$ Y. Seiya, ${ }^{40}$ A. Semenov, ${ }^{14}$ F. Semeria ${ }^{5}$ L. Sexton-Kennedy, ${ }^{16}$ I. Sfiligoi, ${ }^{18}$ M. D. Shapiro, ${ }^{28}$ T. Shears,${ }^{29}$ P. F. Shepard, ${ }^{45}$ D. Sherman,${ }^{21}$ M. Shimojima, ${ }^{53}$ M. Shochet, ${ }^{13}$ Y. Shon ${ }^{57}$ I. Shreyber, ${ }^{35}$ A. Sidoti,${ }^{44}$ A. Sill, ${ }^{16}$ P. Sinervo, ${ }^{32}$ A. Sisakyan, ${ }^{14}$ J. Sjolin, ${ }^{41}$ A. Skiba ${ }^{25}$ A. J. Slaughter,${ }^{16}$ K. Sliwa, ${ }^{54}$ D. Smirnov, ${ }^{36}$ J. R. Smith, ${ }^{7}$ F. D. Snider, ${ }^{16}$ R. Snihur, ${ }^{32}$ M. Soderberg, ${ }^{33}$ A. Soha,${ }^{7}$ S. Somalwar, ${ }^{50}$ V. Sorin, ${ }^{34}$ J. Spalding, ${ }^{16}$ F. Spinella, ${ }^{44}$ P. Squillacioti, ${ }^{44}$ M. Stanitzki ${ }^{58}$ A. Staveris-Polykalas, ${ }^{44}$ R. St. Denis, ${ }^{20}$ B. Stelzer, ${ }^{8}$ O. Stelzer-Chilton, ${ }^{32}$ D. Stentz, ${ }^{37}$ J. Strologas, ${ }^{36}$ D. Stuart, ${ }^{10}$ J. S. Suh, ${ }^{27}$ A. Sukhanov, ${ }^{17}$ K. Sumorok, ${ }^{31}$ H. Sun, ${ }^{54}$ T. Suzuki, ${ }^{53}$ A. Taffard,${ }^{23}$ R. Tafirout, ${ }^{32}$ R. Takashima,${ }^{39}$ Y. Takeuchi,${ }^{53}$ K. Takikawa, ${ }^{53}$ M. Tanaka, ${ }^{2}$ R. Tanaka, ${ }^{39}$ M. Tecchio, ${ }^{33}$ P. K. Teng, ${ }^{1}$ K. Terashi, ${ }^{48}$ S. Tether, ${ }^{31}$ J. Thom, ${ }^{16}$ A. S. Thompson, ${ }^{20}$ E. Thomson, ${ }^{43}$ P. Tipton, ${ }^{47}$ V. Tiwari, ${ }^{12}$ S. Tkaczyk,${ }^{16}$ D. Toback, ${ }^{51}$ K. Tollefson, ${ }^{34}$ T. Tomura, ${ }^{53}$ D. Tonelli, ${ }^{44}$ M. Tönnesmann, ${ }^{34} \mathrm{~S}$. Torre, ${ }^{44} \mathrm{D}$. Torretta, ${ }^{16} \mathrm{~S}$. Tourneur, ${ }^{16} \mathrm{~W}$. Trischuk,${ }^{32} \mathrm{R}$. Tsuchiya, ${ }^{55} \mathrm{~S}$. Tsuno, ${ }^{39} \mathrm{~N}$. Turini, ${ }^{44}$ F. Ukegawa, ${ }^{53}$ T. Unverhau, ${ }^{20}$ S. Uozumi,${ }^{53}$ D. Usynin, ${ }^{43}$ L. Vacavant, ${ }^{28}$ A. Vaiciulis, ${ }^{47}$ S. Vallecorsa, ${ }^{19}$ A. Varganov,${ }^{33}$ E. Vataga, ${ }^{36}$ G. Velev, ${ }^{16}$ G. Veramendi, ${ }^{23}$ V. Veszpremi, ${ }^{46}$ T. Vickey, ${ }^{23}$ R. Vidal, ${ }^{16}$ I. Vila, ${ }^{11}$ R. Vilar, ${ }^{11}$ I. Vollrath, ${ }^{32}$ I. Volobouev, ${ }^{28}$ F. Würthwein, ${ }^{9}$ P. Wagner, ${ }^{51}$ R. G. Wagner, ${ }^{2}$ R. L. Wagner, ${ }^{16}$ W. Wagner, ${ }^{25}$ R. Wallny, ${ }^{8}$ T. Walter, ${ }^{25}$ Z. Wan, ${ }^{50}$ M. J. Wang, ${ }^{1}$ S. M. Wang, ${ }^{17}$ A. Warburton,${ }^{32}$ B. Ward,${ }^{20}$ S. Waschke, ${ }^{20}$ D. Waters,${ }^{30}$ T. Watts ${ }^{50}$ M. Weber,${ }^{28}$ W. C. Wester III, ${ }^{16}$ B. Whitehouse, ${ }^{54}$ D. Whiteson, ${ }^{43}$ A. B. Wicklund, ${ }^{2}$ E. Wicklund, ${ }^{16}$ H. H. Williams,${ }^{43}$ P. Wilson, ${ }^{16}$ B. L. Winer ${ }^{38}$ P. Wittich, ${ }^{43}$ S. Wolbers,${ }^{16}$ C. Wolfe, ${ }^{13}$ S. Worm,${ }^{50}$ T. Wright,${ }^{33}$ X. Wu, ${ }^{19}$ S. M. Wynne, ${ }^{29}$ A. Yagil, ${ }^{16}$ K. Yamamoto, ${ }^{40}$ J. Yamaoka ${ }^{50}$ Y. Yamashita,${ }^{39}$ C. Yang, ${ }^{58}$ U. K. Yang, ${ }^{13}$ W. M. Yao, ${ }^{28}$ G. P. Yeh, ${ }^{16}$ J. Yoh, ${ }^{16}$ K. Yorita, ${ }^{13}$ T. Yoshida, ${ }^{40}$ I. Yu, ${ }^{27}$ S. S. Yu, ${ }^{43}$ J. C. Yun, ${ }^{16}$ L. Zanello, ${ }^{49}$ A. Zanetti, ${ }^{52}$ I. Zaw ${ }^{21}$ F. Zetti, ${ }^{44}$ X. Zhang, ${ }^{23}$ J. Zhou, ${ }^{50}$ and S. Zucchelli ${ }^{5}$

(CDF Collaboration)

\author{
${ }^{1}$ Institute of Physics, Academia Sinica, Taipei, Taiwan 11529, Republic of China \\ ${ }^{2}$ Argonne National Laboratory, Argonne, Illinois 60439, USA \\ ${ }^{3}$ Institut de Fisica d'Altes Energies, Universitat Autonoma de Barcelona, E-08193, Bellaterra (Barcelona), Spain \\ ${ }^{4}$ Baylor University, Waco, Texas 76798, USA \\ ${ }^{5}$ Istituto Nazionale di Fisica Nucleare, University of Bologna, I-40127 Bologna, Italy \\ ${ }^{6}$ Brandeis University, Waltham, Massachusetts 02254, USA \\ ${ }^{7}$ University of California, Davis, California 95616, USA \\ ${ }^{8}$ University of California, Los Angeles, Los Angeles, California 90024, USA \\ ${ }^{9}$ University of California, San Diego, La Jolla, California 92093, USA \\ ${ }^{10}$ University of California, Santa Barbara, Santa Barbara, California 93106, USA \\ ${ }^{11}$ Instituto de Fisica de Cantabria, CSIC-University of Cantabria, 39005 Santander, Spain \\ ${ }^{12}$ Carnegie Mellon University, Pittsburgh, Pennsylvania 15213, USA \\ ${ }^{13}$ Enrico Fermi Institute, University of Chicago, Chicago, Illinois 60637, USA \\ ${ }^{14}$ Joint Institute for Nuclear Research, RU-141980 Dubna, Russia \\ ${ }^{15}$ Duke University, Durham, North Carolina 27708, USA \\ ${ }^{16}$ Fermi National Accelerator Laboratory, Batavia, Illinois 60510, USA \\ ${ }^{17}$ University of Florida, Gainesville, Florida 32611, USA \\ ${ }^{18}$ Laboratori Nazionali di Frascati, Istituto Nazionale di Fisica Nucleare, I-00044 Frascati, Italy \\ ${ }^{19}$ University of Geneva, CH-1211 Geneva 4, Switzerland \\ ${ }^{20}$ Glasgow University, Glasgow G12 8QQ, United Kingdom \\ ${ }^{21}$ Harvard University, Cambridge, Massachusetts 02138, USA \\ ${ }^{22}$ Division of High Energy Physics, Department of Physics, University of Helsinki and Helsinki Institute of Physics, \\ FIN-00014, Helsinki, Finland
}




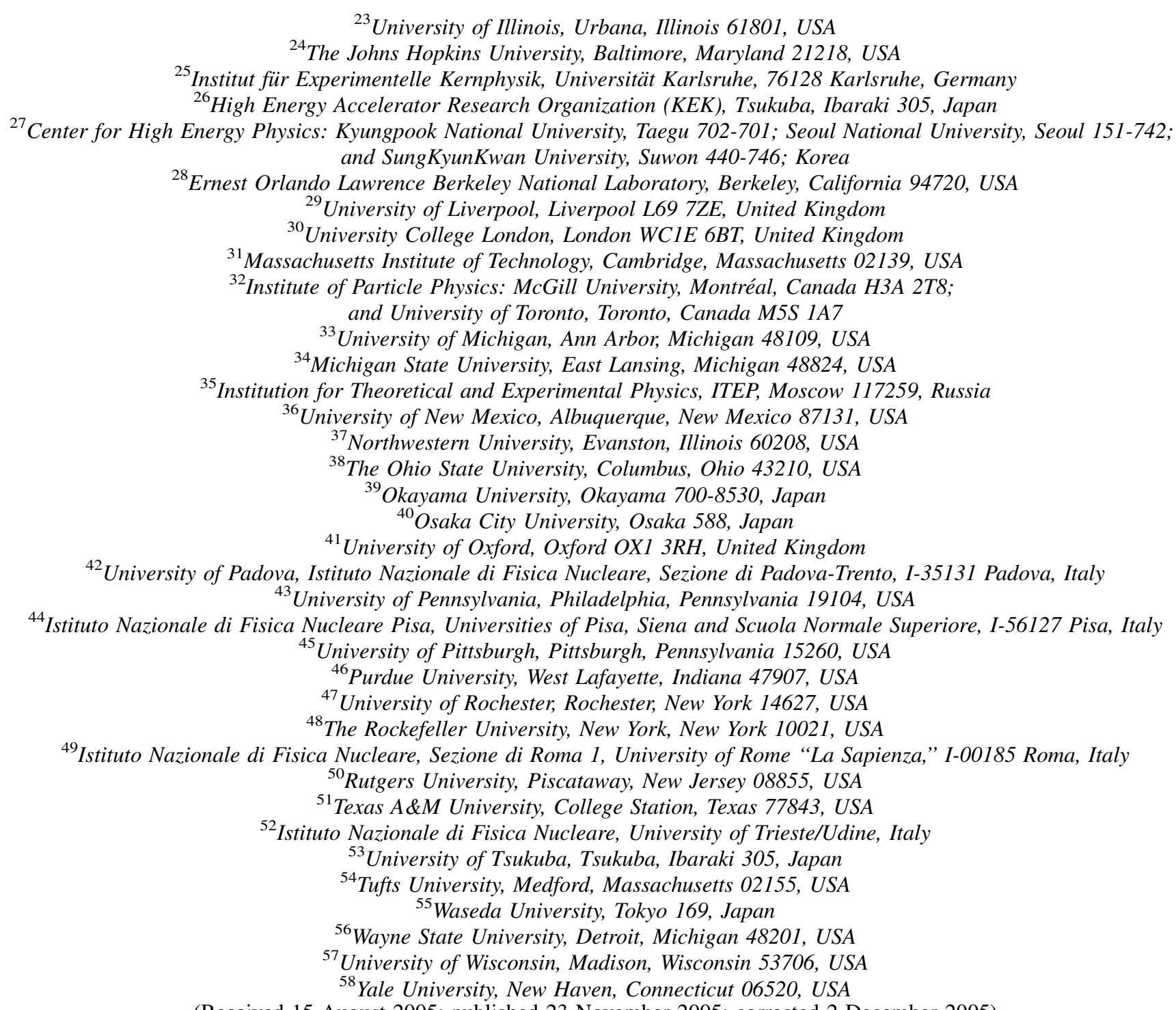

(Received 15 August 2005; published 23 November 2005; corrected 2 December 2005)

\begin{abstract}
We report on a search for $B_{s}^{0} \rightarrow \mu^{+} \mu^{-}$and $B_{d}^{0} \rightarrow \mu^{+} \mu^{-}$decays in $p \bar{p}$ collisions at $\sqrt{s}=1.96 \mathrm{TeV}$ using $364 \mathrm{pb}^{-1}$ of data collected by the CDF II detector at the Fermilab Tevatron Collider. After applying all selection requirements, we observe no candidates inside the $B_{s}^{0}$ or $B_{d}^{0}$ mass windows. The resulting upper limits on the branching fractions are $\mathcal{B}\left(B_{s}^{0} \rightarrow \mu^{+} \mu^{-}\right)<1.5 \times 10^{-7}$ and $\mathcal{B}\left(B_{d}^{0} \rightarrow \mu^{+} \mu^{-}\right)<3.9 \times$ $10^{-8}$ at $90 \%$ confidence level.
\end{abstract}

DOI: 10.1103/PhysRevLett.95.221805

In the standard model (SM), flavor changing neutral current (FCNC) decays are highly suppressed and can occur only through higher order diagrams. The decay rate for the FCNC decay $B_{s}^{0} \rightarrow \mu^{+} \mu^{-}$[1] is proportional to the Cabibbo-Kobayashi-Maskawa matrix element $\left|V_{t s}\right|^{2}$. The rate of $B_{d}^{0} \rightarrow \mu^{+} \mu^{-}$decays is further suppressed by the ratio of $\left|V_{t d} / V_{t s}\right|^{2}$. The SM expectations for these branching fractions are $\mathcal{B}\left(B_{s}^{0} \rightarrow \mu^{+} \mu^{-}\right)=(3.42 \pm$ $0.54) \times 10^{-9}$ and $\mathcal{B}\left(B_{d}^{0} \rightarrow \mu^{+} \mu^{-}\right)=(1.00 \pm 0.14) \times$ $10^{-10}$ [2], which are about 2 orders of magnitude smaller than the current experimental sensitivity. However, new physics contributions can significantly enhance these branching fractions. An observation of these decays at the Tevatron would be unambiguous evidence for physics
PACS numbers: 13.20.He, 12.15.Mm, 12.60.Jv, 13.85.Qk

beyond the SM. The best existing experimental bound on $\mathcal{B}$ is $<4.1 \times 10^{-7}$ [3] $\left(<8.3 \times 10^{-8}[4]\right)$ for $B_{s}^{0}\left(B_{d}^{0}\right) \rightarrow$ $\mu^{+} \mu^{-}$at the $90 \%$ confidence level (C.L.).

In minimal supersymmetric (SUSY) extensions of the SM, additional diagrams involving SUSY particles also contribute to FCNC decay rates and the branching fraction $\mathcal{B}\left(B_{s, d}^{0} \rightarrow \mu^{+} \mu^{-}\right) \propto(\tan \beta)^{6}$, where $\tan \beta$ is the ratio of vacuum expectation values of the two neutral $C P$-even Higgs fields. Large values of $\tan \beta$ enhance the decay rate to a level observable by the Tevatron experiments [5]. For example, increases of 1-3 orders of magnitude are obtained in the minimal $\mathrm{SO}(10)$ models [6], which favor large values of $\tan \beta$. For the minimal flavor violating (MFV) models, $B_{d}^{0} \rightarrow \mu^{+} \mu^{-}$remains suppressed relative to 
$B_{s}^{0} \rightarrow \mu^{+} \mu^{-}$due to $\left|V_{t d} / V_{t s}\right|^{2}$. This may not be true for non-MFV models such as $R$-parity violating SUSY [7], which can produce large enhancements, even for low values of $\tan \beta$, in either or both of the $B_{s}$ and $B_{d}$ FCNC decay rates. Thus, a simultaneous observation of $B_{s, d}^{0} \rightarrow \mu^{+} \mu^{-}$ decays can be important in determining the flavor structure of the new physics. In the absence of an observation, any improvements to the limits can be used to constrain significantly many SUSY models [5-8].

In this Letter, we report on a search for $B_{s}^{0} \rightarrow \mu^{+} \mu^{-}$and $B_{d}^{0} \rightarrow \mu^{+} \mu^{-}$decays using $364 \mathrm{pb}^{-1}$ of data collected by the upgraded Collider Detector at Fermilab (CDF II). This data set includes $171 \mathrm{pb}^{-1}$ of data from our previous measurement [9]. We significantly improve the sensitivity of the search over our former analysis by doubling our data sample, extending the muon acceptance, and using a likelihood ratio technique for signal and background separation. The limits we present here are the most stringent to date and supersede our previous results.

The CDF II detector is described in detail in Ref. [10]. The inner tracking system is composed of a silicon microstrip detector (SVX II) [11] surrounded by an open-cell wire drift chamber (COT) [12]. These tracking detectors are immersed in a $1.4 \mathrm{~T}$ magnetic field and measure $p_{T}$, charged particle momentum in the plane transverse to the beam line. Four layers of planar drift chambers (CMU) [13] detect muon candidates with $p_{T}>1.4 \mathrm{GeV} / c$ and provide coverage in the pseudorapidity range $|\eta|<0.6$, where $\eta=-\ln \left(\tan \frac{\theta}{2}\right)$ and $\theta$ is the angle of the track with respect to the beam line. The central muon extension (CMX) consists of conical sections of drift tubes and extends the coverage to $0.6<|\eta|<1.0$ for muon candidates with $p_{T}>2.0 \mathrm{GeV} / c$.

The data used in this analysis are selected by two classes of dimuon triggers: for the CMU-CMU $(U-U)$ triggers, both muon candidates are reconstructed in the CMU chambers, while for the CMU-CMX $(U-X)$ triggers, one of the muon candidates is reconstructed in the CMX chambers. The inclusion of the $U-X$ trigger increases the signal acceptance by about $50 \%$. The details of the trigger system and selection requirements can be found in Refs. $[9,10]$. Since they have different sensitivities, we treat $U-U$ and $U-X$ channels separately, combining the results at the end.

The offline reconstruction begins by identifying two muon candidates of opposite charge which satisfy the online dimuon trigger requirements. To avoid regions of rapidly changing trigger efficiency, we omit CMU (CMX) muon candidates with $p_{T}<2(2.2) \mathrm{GeV} / c$. The random combinatoric backgrounds are suppressed by requiring the vector sum of the muon transverse momenta to be $\left|\vec{p}_{T}^{\mu \mu}\right|>$ $4 \mathrm{GeV} / c$. The remaining pairs of muon tracks are then refit, under the constraint that they come from the same three-dimensional (3D) space point, and are required to satisfy vertex fit quality criteria. The 3D decay length is given by $L_{3 \mathrm{D}}=\vec{L} \cdot \vec{p}^{\mu \mu} /\left|\vec{p}^{\mu \mu}\right|$, where $\vec{L}$ is the displacement vector from the primary to the dimuon vertex. The primary vertex is determined using a constrained vertex fit of all tracks in the event, excluding the $\mu^{+} \mu^{-}$pair and other secondary decay tracks. For each $B$ candidate, we estimate the proper decay time $\tau=M_{\mu \mu} L_{3 \mathrm{D}} /\left|\vec{p}^{\mu \mu}\right|$, where $M_{\mu \mu}$ is the invariant mass and $\vec{p}^{\mu \mu}$ is the momentum vector of the dimuon system. Additional background is reduced by demanding $L_{3 \mathrm{D}}<1.0 \mathrm{~cm}$ (to remove poorly reconstructed tracks), the uncertainty on $L_{3 \mathrm{D}}$ to be less than $150 \mu \mathrm{m}$, and $2 \sigma_{\lambda}<\lambda<0.3 \mathrm{~cm}$, where $\lambda=c \cdot \tau$ and $\sigma_{\lambda}$ is the total uncertainty on $\lambda$. There are 22459 (14305) dimuon candidates that fulfill all the above trigger and offline reconstruction requirements in the $U-U(U-X)$ channel. At this stage, the data sample is dominated by random combinatoric background.

We model the signal $B_{s, d}^{0} \rightarrow \mu^{+} \mu^{-}$decays using the PYTHIA Monte Carlo (MC) program [14]. The PYTHIA events are passed through a full detector simulation and satisfy the same requirements as data. The $p_{T}$ spectrum of the $B$ hadron in the MC sample is weighted to match the measurement from Ref. [10]. The signal MC samples are used primarily for analysis optimization and for estimating the efficiency of selection requirements.

For the final event selection, we use the following four discriminating variables: $M_{\mu \mu}, \lambda$, the 3D opening angle $\Delta \Theta$ between vectors $\vec{p}^{\mu \mu}$ and $\vec{L}$, and the $B$-candidate track isolation (I) [15]. We exploit the long lifetime of $B$ mesons to reject prompt combinatoric background with the decay length $(\lambda)$ requirement. Additional combinatoric background and partially reconstructed $B$ hadrons are removed with the pointing angle requirement. Since $b$-quark fragmentation is hard, $B$ hadrons carry most of the transverse momentum of the $b$ quark and, thus, are isolated. We use the variable $I$ to enhance the heavy flavor content of the sample and also to reject partially reconstructed $B$ hadrons, which are less isolated. Figure 1 compares the distributions of these variables for data (which is backgrounddominated) to MC signal events. Based on the observed distributions, we apply two additional loose requirements, $I>0.5$ and $\Delta \Theta<0.7 \mathrm{rad}$, to further reject background while maintaining $92 \%$ of signal efficiency. We collectively refer to all the selection requirements applied up to this point as the "baseline" requirements. In the data, 6242 (4908) events survive the baseline requirements in the $U-U(U-X)$ sample.

To enhance signal and background separation, we construct a multivariate likelihood ratio based on the input

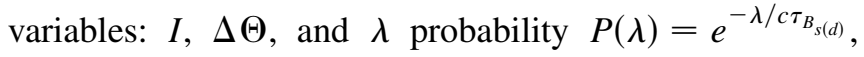
where $\tau_{B_{s(d)}}$ is the world average $B_{s(d)}$ lifetime. We use the $P(\lambda)$ variable instead of $\lambda$ in constructing the likelihood ratio, because the $P(\lambda)$ distribution is nearly flat and, therefore, better behaved in the likelihood. We define the likelihood ratio to be

$$
L_{R}=\frac{\prod_{i} \mathbf{P}_{s}\left(x_{i}\right)}{\prod_{i} \mathbf{P}_{s}\left(x_{i}\right)+\prod_{i} \mathbf{P}_{b}\left(x_{i}\right)},
$$



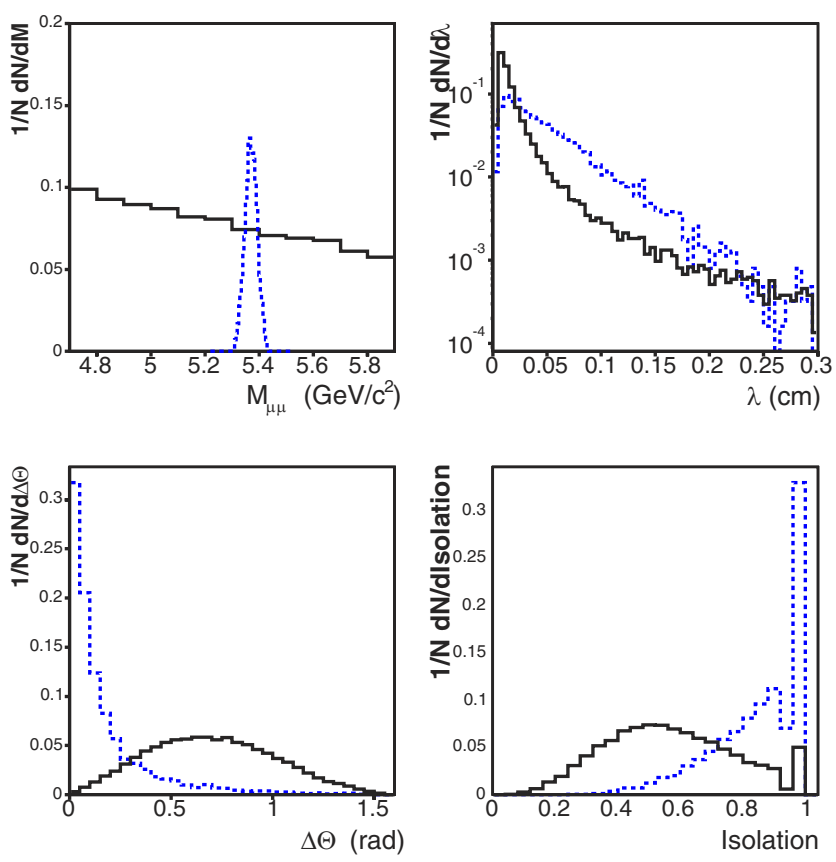

FIG. 1 (color online). Distributions of the discriminating variables for data (dominated by background) shown in the solid histogram and signal $B_{s}^{0} \rightarrow \mu^{+} \mu^{-}$events shown in the dashed histogram.

where $x_{1}=I, x_{2}=\Delta \Theta, x_{3}=P(\lambda)$, and $\mathbf{P}_{s(b)}\left(x_{i}\right)$ is the probability that a signal (background) event has an observed $x_{i}$. The probability distributions for the signal events are obtained from the signal MC samples and the background distributions are taken from the data sidebands (defined below). The resulting $L_{R}$ distributions for the signal and background events are shown in Fig. 2.

Although a subset of the data was used previously [9], we adopt an optimization strategy that uses only events in

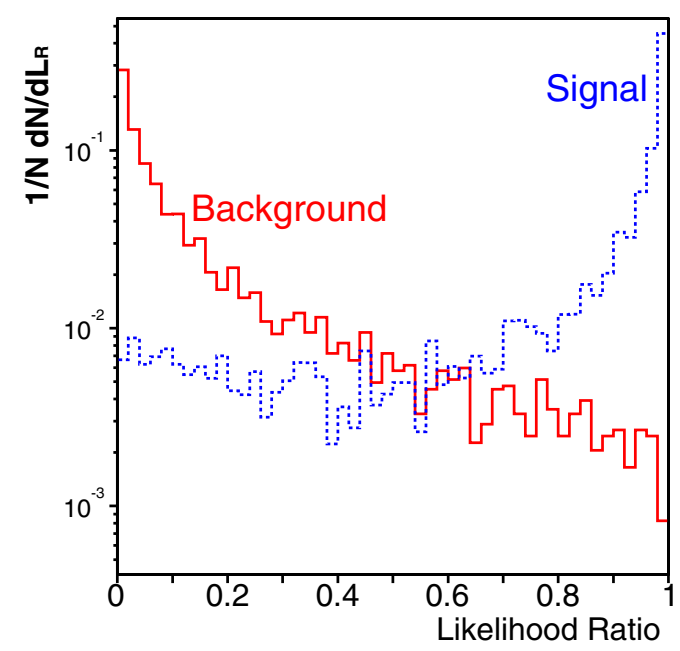

FIG. 2 (color online). The likelihood ratio distribution for signal (dashed line) from PYTHIA MC samples and background (solid line) from data sidebands in the $U-U$ channel. Similar shapes are observed in the $U-X$ channel. the data sidebands in order to avoid biases in our choice of final selection criteria. The search window is defined by $5.169<M_{\mu \mu}<5.469 \mathrm{GeV} / c^{2}$, corresponding to approximately \pm 6 times the two-track invariant mass resolution, which is estimated to be $\sigma_{M} \approx 24 \mathrm{MeV} / c^{2}$. The sideband regions $4.669<M_{\mu \mu}<5.169 \mathrm{GeV} / c^{2}$ and $5.469<$ $M_{\mu \mu}<5.969 \mathrm{GeV} / c^{2}$ are used to estimate the background in the signal region and for extensive analysis cross-checks.

The $B_{s}^{0} \rightarrow \mu^{+} \mu^{-}$branching fraction is obtained by normalizing to the number of $B^{+} \rightarrow J / \psi K^{+} \rightarrow \mu^{+} \mu^{-} K^{+}$ decays collected by the same trigger. The $B^{+} \rightarrow J / \psi K^{+}$ mode is reconstructed using the same baseline requirements as the signal mode [16] but including an additional $p_{T}>1 \mathrm{GeV} / c$ requirement on the kaon candidate. The upper limit on the branching fraction is given by

$$
\begin{aligned}
\mathcal{B}\left(B_{s}^{0} \rightarrow \mu^{+} \mu^{-}\right)^{90 \% \text { C.L. }=}= & \frac{N_{B_{s}^{0}}^{90 \%}}{N_{B^{+}}} \frac{\alpha_{B^{+}}}{\alpha_{B_{s}^{0}}} \frac{\epsilon_{B^{+}}^{\text {base }}}{\epsilon_{B_{s}^{0}}^{\text {base }}} \frac{1}{\epsilon_{B_{s}^{0}}^{L_{R}}} \\
& \times \frac{f_{u}}{f_{s}} \mathcal{B}\left(B^{+} \rightarrow J / \psi K^{+}\right),
\end{aligned}
$$

where $N_{B_{s}^{0}}^{90 \%}$ is the number of $B_{s}^{0} \rightarrow \mu^{+} \mu^{-}$decays at the 90\% C.L. for $N$ observed and $N_{b}$ expected background events. The value of $N_{B_{s}^{0}}^{90 \%}$ is estimated using the Bayesian approach of Ref. [17], assuming a flat prior for the $\mathcal{B}\left(B_{s}^{0} \rightarrow\right.$ $\mu^{+} \mu^{-}$), and incorporating Gaussian uncertainties into the limit. The number of reconstructed $B^{+} \rightarrow J / \psi K^{+}$candidates, $N_{B^{+}}$, determined from the data using sideband subtraction is $1785 \pm 60$ for the $U-U$ and $696 \pm 39$ for the $U-X$ channel. The parameter $\alpha_{B_{s}^{0}}\left(\alpha_{B^{+}}\right)$is the trigger acceptance and $\epsilon_{B^{0}}^{\text {base }}\left(\epsilon_{B^{+}}^{\text {base }}\right)$ is the efficiency of the baseline requirements for the signal (normalization) mode. We apply the likelihood ratio requirement only to the signal mode, and, therefore, the efficiency of the likelihood ratio $\epsilon_{B_{s}^{0}}^{L_{R}}$ appears only in the denominator of Eq. (2). We use the fragmentation ratio $f_{u} / f_{s}=3.71 \pm 0.41$ [18]. The branching fraction $\mathcal{B}\left(B^{+} \rightarrow J / \psi K^{+} \rightarrow \mu^{+} \mu^{-} K^{+}\right)=$ $(5.88 \pm 0.26) \times 10^{-5}$ is obtained from Ref. [17]. The expression for $\mathcal{B}\left(B_{d}^{0} \rightarrow \mu^{+} \mu^{-}\right)$is derived by replacing $B_{s}^{0}$ with $B_{d}^{0}$ and the fragmentation ratio with $f_{u} / f_{d}=1$.

The expected number of background events is estimated by extrapolating the number of sideband events passing the baseline requirements to the signal window and then scaling by the expected rejection factor $\kappa$ for a given $L_{R}$ requirement. The parameter $\kappa$ is determined from the background $L_{R}$ distribution, which we generate by randomly sampling the $\lambda, \Delta \Theta$, and $I$ distributions from the data sidebands to improve statistical precision on $\kappa$. The relative uncertainty on $N_{b}$ is $\pm 15 \%$ ( $\pm 19 \%$ ) for the $U-U$ $(U-X)$ channel. The dominant contribution comes from the limited statistics of the input distributions used to generate the background $L_{R}$ distribution.

We have cross-checked our background estimate procedure using control samples from the data: like sign $\mu^{ \pm} \mu^{ \pm}$ 
events, $\mu^{+} \mu^{-}$events with $\lambda<0$, and a fake-muon enhanced $\mu^{+} \mu^{-}$sample in which we demand at least one muon candidate fail the muon quality requirements. We compare our background predictions to the number of events observed in the search window for a wide range of $L_{R}$ requirements. No statistically significant discrepancies are observed. For example, requiring $L_{R}>0.99$ and summing over all control samples, we predict 3.8 (4.0) background events and observe 4 (3) in the $U-U(U-X)$ channel. In addition, we have considered background contributions from $B_{s, d}^{0} \rightarrow h^{+} h^{-}$, where $h^{ \pm}=\pi^{ \pm}$or $K^{ \pm}$, in the signal window and determined that the contribution from those decays is negligible.

The acceptance ratio $\alpha_{B^{+}} / \alpha_{B_{s, d}^{0}}$ obtained from the signal MC sample is $0.297 \pm 0.020(0.191 \pm 0.013)$ for the $U-U$ $(U-X)$ channel. The uncertainty includes contributions from systematic variations of the modeling of the $B$-hadron $p_{T}$ spectrum and longitudinal beam profile and from the statistics of the MC sample.

The quantity $\epsilon^{\text {base }}$ includes the trigger and offline reconstruction efficiencies. The trigger efficiency is determined from inclusive data samples unbiased with respect to the triggers used here. The ratio of $B^{+}$to $B_{s}^{0}$ trigger efficiencies is measured to be less than $0.1 \%$ away from unity. We evaluate the single track COT, SVX II, and muon efficiencies using a data sample of inclusive $J / \psi \rightarrow \mu^{+} \mu^{-}$decays collected on single-muon triggers. The relevant doubletrack efficiencies are computed by convolution with $B_{s}^{0} \rightarrow$ $\mu^{+} \mu^{-}$and $B^{+} \rightarrow J / \psi K^{+}$MC events surviving the trigger requirements. The offline reconstruction efficiency between signal and normalization mode also largely cancels in the ratio with the exception of the kaon efficiency from the $B^{+}$decay. Last, we obtain the efficiency of the remaining baseline requirements from the signal MC sample and cross-check the results by comparing $B^{+}$data and MC samples. Combining all effects, we find $\epsilon_{B^{+}}^{\text {base }} / \epsilon_{B^{0}}^{\text {base }}=$ $0.920 \pm 0.034(0.915 \pm 0.034)$ for the $U-U(U-X)$ channel. The uncertainty is dominated by systematic uncertainties accounting for kinematic differences between $J / \psi \rightarrow \mu^{+} \mu^{-}$and $B_{s, d}^{0} \rightarrow \mu^{+} \mu^{-}$decays.

The efficiency of the likelihood requirement $\epsilon_{B_{s, d}^{0}}^{L_{R}}$ is estimated from the signal MC sample. The efficiency varies from about $70 \%$ for $L_{R}>0.90$ to $35 \%$ for $L_{R}>$ 0.99 . We assign a relative systematic uncertainty of $\pm 5 \%$ to both $U-U$ and $U-X$ channels based on comparisons of $B^{+} \rightarrow J / \psi K^{+} \mathrm{MC}$ and data samples.

We optimize the analysis based on the a priori expected $90 \%$ C.L. upper limit on $\mathcal{B}\left(B_{s, d}^{0} \rightarrow \mu^{+} \mu^{-}\right)$. The expected limit for a given set of optimization requirements is computed by summing the $90 \%$ C.L. limits over all possible observations $N$, weighted by the corresponding Poisson probability when expecting $N_{b}$. We scan over a range of $L_{R}$ requirements and determine the optimal value to be $L_{R}>0.99$. With the optimized selection requirements, the expected number of background events is the same in the

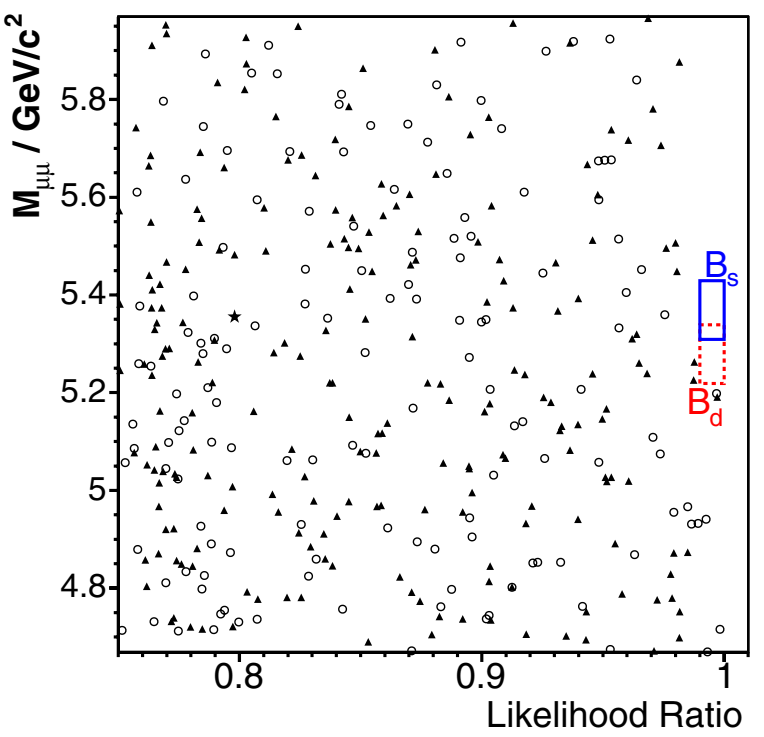

FIG. 3 (color online). The $\mu^{+} \mu^{-}$invariant mass distribution versus likelihood ratio for events satisfying baseline requirements for $U-U$ (solid triangles) and $U-X$ (open circles) channels. The $B_{s}^{0}$ (solid box) and $B_{d}^{0}$ (dashed box) signal regions are also shown. The one candidate event observed in the previous analysis is highlighted with a star symbol (at $L_{R} \approx 0.8$ ).

$B_{s}^{0}$ and $B_{d}^{0}$ search windows. Within the signal region of $\pm 60 \mathrm{MeV} / c^{2}\left( \pm 2.5 \sigma_{M}\right)$ about the world average $B_{s}^{0}$ or $B_{d}^{0}$ mass [17], $N_{b}$ is $0.81 \pm 0.12[0.66 \pm 0.13]$, and the $B_{s}^{0}$ single-event sensitivity is $(1.0 \pm 0.2) \times 10^{-7}[(1.5 \pm$ $\left.0.3) \times 10^{-7}\right]$ for the $U-U[U-X]$ channel. Using these criteria, we observe no events inside either the $B_{s}^{0}$ or $B_{d}^{0}$ signal box as shown in Fig. 3. The one candidate event that survived in the previous analysis [9] has an $L_{R}$ value of $\approx$ 0.8 and fails the selection requirements here. Using Eq. (2) and combining the $U-U$ and $U-X$ channels, taking into account the correlated uncertainties, we derive $90 \%$ (95\%) C.L. limits of $\mathcal{B}\left(B_{s}^{0} \rightarrow \mu^{+} \mu^{-}\right)<1.5 \times 10^{-7} \quad(2.0 \times$ $\left.10^{-7}\right)$ and $\mathcal{B}\left(B_{d}^{0} \rightarrow \mu^{+} \mu^{-}\right)<3.9 \times 10^{-8}\left(5.1 \times 10^{-8}\right)$. The new limits improve the previous limits $[3,4]$ by a factor of 2 and can be used to reduce the allowed parameter space of a broad spectrum of SUSY models [6-8].

We thank the Fermilab staff and the technical staffs of the participating institutions for their vital contributions. This work was supported by the U.S. Department of Energy and National Science Foundation; the Italian Istituto Nazionale di Fisica Nucleare; the Ministry of Education, Culture, Sports, Science and Technology of Japan; the Natural Sciences and Engineering Research Council of Canada; the National Science Council of the Republic of China; the Swiss National Science Foundation; the A. P. Sloan Foundation; the Bundesministerium für Bildung und Forschung, Germany; the Korean Science and Engineering Foundation and the Korean Research Foundation; the Particle Physics and Astronomy Research Council and the Royal Society, U.K.; the Russian Foundation for Basic Research; the Comisión Interministerial de Ciencia y Tecnología, Spain; in part by the European 
Community's Human Potential Programme under Contract No. HPRN-CT-2002-00292; and the Academy of Finland.

[1] Throughout this paper, inclusion of charge conjugate modes is implicit.

[2] G. Buchalla and A. J. Buras, Nucl. Phys. B400, 225 (1993); A. J. Buras, Phys. Lett. B 566, 115 (2003).

[3] V. Abazov et al. (D0 Collaboration), Phys. Rev. Lett. 94, 071802 (2005).

[4] B. Aubert et al. (BABAR Collaboration), Phys. Rev. Lett. 94, 221803 (2005).

[5] S. Choudhury and N. Gaur, Phys. Lett. B 451, 86 (1999); K. S. Babu and C. Kolda, Phys. Rev. Lett. 84, 228 (2000).

[6] R. Dermisek et al., J. High Energy Phys. 04 (2003) 037; D. Auto et al., J. High Energy Phys. 06 (2003) 023; T. Blazek et al., Phys. Lett. B 589, 39 (2004).

[7] R. Arnowitt et al., Phys. Lett. B 538, 121 (2002).

[8] H. Baer et al., J. High Energy Phys. 07 (2002) 050; S. Baek, P. Ko, and W. Y. Song, Phys. Rev. Lett. 89, 271801 (2002); H. Logan and U. Nierste, Phys. Lett. B 566, 115 (2003); A. Dedes and B. Huffman, Phys. Lett. B
600, 261 (2004); G. L. Kane, C. Kolda, and J. E. Lennon, hep-ph/0310042.

[9] D. Acosta et al. (CDF Collaboration), Phys. Rev. Lett. 93, 032001 (2004).

[10] D. Acosta et al. (CDF Collaboration), Phys. Rev. D 71, 032001 (2005).

[11] A. Sill et al., Nucl. Instrum. Methods Phys. Res., Sect. A 447, 1 (2000).

[12] T. Affolder et al., Nucl. Instrum. Methods Phys. Res., Sect. A 526, 249 (2004).

[13] G. Ascoli et al., Nucl. Instrum. Methods Phys. Res., Sect. A 268, 33 (1988).

[14] T. Sjöstrand et al., Comput. Phys. Commun. 135, 238 (2001).

[15] The $B$-candidate isolation is defined as $I=\left|\vec{p}_{T}^{\mu \mu}\right| /$ $\left(\sum_{i} p_{T}^{i}+\left|\vec{p}_{T}^{\mu \mu}\right|\right)$, where the sum is over all tracks with $\sqrt{\Delta \eta^{2}+\Delta \phi^{2}} \leq 1 ; \Delta \phi$ and $\Delta \eta$ are, respectively, the azimuthal angle and pseudorapidity of track $i$ with respect to $\vec{p}^{\mu \mu}$. Also see V. Krutelyov, Ph.D. thesis, Texas A\&M University, 2005 (unpublished).

[16] We use only the two muon tracks in the $B^{+}$vertex fit.

[17] S. Eidelman et al., Phys. Lett. B 592, 1 (2004).

[18] $f_{x}$ is the fraction of weakly decaying $B_{x}$ hadron in $b$ quark fragmentation. We use values from Ref. [17] and take into account the correlations between $f_{s}$ and $f_{u}$. 